Rev. Saúde públ., S. Paulo

9:401-7, 1975

\title{
PERSPECTIVAS DA PARTICIPAÇÃO DOS HOSPITAIS GERAIS NA ASSISTENCIA AOS DOENTES DE HANSENIASE*
}

GuIMarães, C. - Perspectivas da participação dos hospitais gerais na assistência aos doentes de hanseníase. Rev. Saúde públ., S. Paulo, 9:401-7, 1975.

RESUMo: Uma sintese histórica demonstra que o hospital tem voltado sua assistência para os aspectos da medicina curativa, para o que muito têm contribuido os progressos de diagnóstico e terapêutica. Os lazaretos e sua ver. são posterior, os hospitais de isolamento, por sua vez, colaboraram para que o hospital geral se mantivesse afastado, até recentemente, da assistência às doenças infecto-contagiosas e até o presente, ao mal de Hansen, sendo referidas as excecões de hospitais escolas de São Paulo. destacada a importancia da educação do corpo clinico, administração e pacientes dos hospitais, bem como do público em geral, como meio de tornar factivel, a curto prazo, a integração da assistência dos portadores de hanseniase nos hospitais gerais. Como solução, é proposto o enfoque sistêmico, sendo ressaltado o valor da hanseniase ser considerada como componente normal da problemática geral de saúde e não como um aspecto isolado. As vantagens da adoção desse enfoque são apontadas, situando o hanseniano na multidão assistida pelos hospitais, de forma a torná-lo um paciente qualquer.

UNITERMOS: Hospitais gerais. Assistência médica. Hanseniase. Saúde. Integração.

0 aparecimento do hospital geral como centro de diagnóstico e tratamento deu-se nos últimos anos do século passado e primeiros do presente. Foi conseqüência de uma longa evolução caracterizada por três aspectos: a) domínio sobre a dor devido a C. W. Long $(1815 / 78), \mathrm{H}$. Wells (1815/48), W. T. G. Morton (1819/68) e J. Y. Simpson $(1811 / 70)$; b) o conhecimento sobre a origem e combate às infecções devido a A. Gordon (1752/99), R. Collins $(1801 / 35)$, I. Semmelweiss $(1818 / 47)$, L. Pasteur $(1822 / 912)$ e c) a descoberta da moderna diagnose em la- boratório, onde se destacaram R. Koch (1843/910) e P. Erlich (1854/915) e a visualização do interior do corpo humano possibilitada pelos raios-X descobertos por W. K. Roentgen $(1845 / 923)^{3,5,18}$.

0 movimento da assistência à saúde pública iniciado na metade do século XIX, na Inglaterra - onde se salientou $\mathrm{E}$. Chadwick $(1800 / 90)^{13}-$ e difundido na Europa encontrou, já no início deste século, quem quisesse vincrlá-lo ao hospital. 0 "Report of Royal Commission on Poor Law 1905-9", na Inglaterra, já inseria um depoimento que "aconselhava a

- Apresentado no Seminário de Diretores de Hospitais de Hansenologia, Bauru, Sanatório Aimorés, 29 e 30 de Junho de 1973.

* Do Departamento de Prática de Saúde Pública da Faculdade de Saúde Pública da USP - Av. Dr. Arnaldo, 715 - São Paulo, SP - Brasil e do Departamento de Técnica Hospitalar da Secretaria de Saúde do Estado de São Paulo. 
GUIMARAES, C. - Perspectivas da participação dos hospitais gerais na assistência aos doentes de hanseniase. Rev. Saúde públ., S. Paulo, 9:401-7, 1975.

reunião dos serviços de saúde pública e dos serviços médicos da Lei dos Pobres sob uma nova autoridade sanitária" ${ }^{1}$. Em Toronto. Canadá. em 1910, foi iniciada estreita colaboração entre os centros de saúde e os hospitais ? ${ }^{7}$ O Partido Trabalhista Inglês, em 1918, publicou um documento onde adrogava. não só a transferência da administração dos centros de saúde para os hospitais, de forma a encorajar a cooperação entre medicina curativa e preventiva, como também, pioneiramente, estabelecia o hospital como um centro ao redor do qual deveria gravitar a assistência à saúde ${ }^{9}$. H. M. Biggs, como diretor do "New York State Department of Health", no relatório anual desse órgão, em 1919, dizia que "seu principal propósito fora o de trazer todas as atividades de saúde de uma comunidade para um mesmo teto a fim de assegurar uma efetiva cooperação e coordenação entre os vários ramos de trabalho da saúde pública, aumentando, assim, a eficiência, reduzindo o custo da administração de cada um e tornando os recursos financeiros mais disponíveis para a administração real do trabalho" "15. E finalmente, o famoso "Dawson of Penn Report" ao Parlamento Britânico sobre a provisão dos serriços médicos e associados, já propunha o "atualíssimo" hospital-ınidade sanitária ${ }^{10}$.

Esses cunceitos, hoje almejados por năo poucos, só tiveram seus princípios firmados em 1933, por ocasião do IV Congresso da "Federation Internationale des Hopitaux", em Knocke sur Mer, Bélgica. Alter " (alemão). Sand (belga), Gruschka ${ }^{11}$ (checo) e Parrisot ${ }^{12}$ e Sarraz-Bournet ${ }^{14}$ (franceses), vozes das mais conceituadas, na época, sobre medicina social, estabeleceram os postulados da integração, hierarquização e regionalização dos órgãos de saúde que mais tarde foram comprovados e que situavam o hospital como o Centro de Saúde da comunidade.

A Segunda Guerra Mundial veio interferir negativamente para que tais con- ceitos evoluissem, pois seu progresso rápido dependia da conjugação do esforço e do pensamento dos luminares europeus no campo da saúde pública, bem como da troca de experiências e da análise conjunta. Os resultados espetaculares da terapêutica, como a quimioterapia e os antibióticos, o arrojo triunfante da cirurgia, apoiada pela nova tecnologia da anestesia, ao lado de uma diagnose cada dia mais perfeita, acrescentando aos raios- $\mathrm{X}$ a rotina dos traçados das correntes elétricas emanadas do miocárdio e da córtex cerebral, E.C.G. e E.E.G. vieram caracterizar ainda mais o hospital como um templo da medicina curativa, relegando ao campo das discussóes apenas seu papel axial na assistência à saúde. Merecem destaque as exceções havidas e, no Brasil, a obra pioneira do antigo Serviço Especial da Saúde, atual Fundação SESP que, já em 1942, instituia seus hospitais-unidades sanitárias em Santarém e Breves ${ }^{8}$.

É dentro desse conceito de templo da medicina curativa que o hospital brasileiro vive até o presente, onde as exceções vêm confirmar a regra. Tem sido uma ilha, ou melhor dito, um castelo medieval, que pouco ou nenhum liame mantém com as outras instituições de assistência médica em particular e, principalmente, com a estrutura oficial representada pela rede de centros de saúde.

É nesse contexto de assistência desintegrada que devem ser analisadas as perspectivas do hospital geral receber pacientes acometidos de doenças tranemissíveis, destacando-se a hanseníase.

Pragmaticamente deve ser recordado que os lazaretos foram instituições seculares que sempre protegeram os hospitais gerais dos casos declarados de determinadas doenças infecto-contagiosas temidas, como a peste, a varíola e a hanseníase. A sua existência é bem anterior ao seu nome; este nasceu somente no século XV, devido a Igreja de Santa Maria de Nazaré, em Veneza, ter sido usada como hos- 
GLIMARães, C. - Perspectivas da participação dos hospitais gerais na assistência aos doentes de hanseniase. Rev. Saúde puibl., S. Paulo, 9:401-7, 1975.

pital para acolher pacientes com peste. (O "in" inicial foi substituido por "l", dada a semelhança com lazareto, de lázaro ${ }^{17}$ ). No século passado os hospitais de isolamento assumiram esse papel, sendo exemplos, no Brasil. o antigo Hospital de Isolamento. em São Paulo - atual Hospital Emílio Ribas - e o Hospital São Sebastião, no Rio de Janeiro. Os hospitais gerais só morosamente foram participando da assistência aos pacientes de doenças infecto-contagiosas.

Parece terem sido o Hospital das Clínicas da Faculdade de Medicina de São Paulo e a Santa Casa de Misericórdia de Santos os introdutores das unidades para internação de portadores de doenças infecto-contagiosas integradas no corpo principal do hospital. Até então. os resquícios não são raros, eram os doentes internados em pavilhões isolados do restante do nosocômio, sendo, geralmente, privados dos recursos proporcionados às demais unidades de internação.

$O$ início do recebimento de portadores de hanseníase ocorreu bem mais tarde. Em 1958, no referido Hospital das Clínicas, na Clínica Dermatológica, foram, ao que se sabe, internados os primeiros casos de hanseníase em hospital geral. 0 motivo da internação originou-se da pesquisa dos efeitos da Anfetericina-B (Squibb) na hanseníase, realizada por Sampaio *, então assistente da Clínica. A conseqüência da internação foi uma ação de protesto do então diretor do Departamento da Profilaxia da Lepra do Estado, que teve como resultado ser reconhecido o direito, quer pelo Conselho de Administração do Hospital, como pelo Coverno do Estado, de serem internados casos de hanseníase no Hospital Escola. Sampaio, ao pleitear a internação junto à Clínica Dermatológica, o fez, sabendo que hospitais de ensino americanos internavam pacientes com a mesma entidade nosológica: casos na enfermaria geral do Belevue Hospital em
New York e no Warren Hospital. em Rochester, esse último vinculado à Mayo Clinic.

Refere Sampaio * que, inicialmente, foram tomadas todas as medidas peculiares à hospitaliazção de um paciente portador de doença infecto-contagiosa. sendo utilizada a mais rigorosa técnica. Com o decorrer dos dias e do tempo, todas essas medidas foram atendidas até o paciente ter livre trânsito pela unidade e não mais haver preconceito por parte do pessoal do hospital ou dos outros pacientes.

Em 1967, Rotberg* , professor de Clínica Dermatológica da Escola Paulista de Medicina, iniciava a internação de portadores de hanseníase no Hospital São Paulo, para fins de ensino. O início difícil. inclusive com denúncias ao então Departamento de Profilaxia da Lepra do Estado. cedeu Jugar a um clima de perfeita compreensão e total colaboração, após a enfermagem ser esclarecida sobre os atuais conceitos que regem a hanseniologia.

Em 1970, como sonsultor a curto prazo da Organização Panamericana da Saúde, na Guiana. vimos serem atendidos no ambulatório do Mahaica Hospital (224 leitos), o hospital para hanseniologia do país, pessoas da comunidade próxima não portadoras de hanseníase e/ou sem qualquer relação familiar com algum portador. $O$ ambulatório ministrava-lhes, inclusire, injeções e dispensava curativos utilizando-se de material descartável. Esse é um exemplo de hospital especializado atender a toda a população no campo pertinente ao ambulatório de hospital geral. O contrário, também, foi constatado: o Georgetown Hospital, na capital, - maior hospital do pais (962 leitos), bem como o New Amsterdan Hospital, o segundo em tamanho e importância (250 leitos), o Suddie Hospital, o terceiro em capacidade (103 leitos) e o Bartica Hospital (40 leitos) mantinham serviços ambulatoriais para hanseníase.

* Comunicasão pessoal. 
GUIMARAES, C. - Perspectivas da participação dos hospitais gerais na assistencla aos doentes de hanseníase. Rev. Saúde públ., S. Paulo, 9:401-7, 1975.

As eventualidades que podem ser catalogadas no atendimento de pacientes portadores de hanseníase, em hospital geral são:

a) intercorrências estranhas, requerendo recursos assistenciais próprios ao hospital geral;

b) tratamento reparador de lesões originárias de hanseníase;

c) surto reacional de hanseníase e

d) uso dos serviços de diagnóstico e tratamento do hospital geral, sempre que necessários.

0 tratamento da hanseníase em regime de internação em hospital geral esta excluído; esse hospital exige dinamizaçáo de seus leitos, näo oterecendo, tambem, ambiente apropriado à prolongada permanéncia ao paciente, aado que a doença não o obriga a manter-se no leito.

Se não é ditícil arrolar e classificar as ocasiões nas quais os hospitais gerais poderiam assistir ao paciente portador de hanseníase, também não o é dizer, aprioristicamente, que, na atual conjuntura, é ditícil tal assistencia ser factivel, como rotina. Us óbices são evidentes e conhecidos de todos; não o tossem e taltariam motivos para a adição do neologismo "hanseníase" e seus derivados, subsitutivos de termos mais antigos que o proprio vernáculo. Yor esse motıvo, deixarāo de ser abordadas as razóes pelas quais os hospitals gerais se mantèm marginalizados da assistencia aos hansenianos. entretanto, é oportuno registrar que a ausencia de comunicação tem sido um dos tatores responsáveis pela manutenção dos preconceitos que alimentam a marginalização. Somente uma campanha pertinaz, planejada e adequadamente implementada poderia remover, paulatinamente, as barreiras que separam os hospitais gerais dos atuais conceitos epidemiológicos da hanseníase. So- mente a comunicação possibilitaria diminuir e, subseqüentemente, afastar os preconceitos que alinham e vinculam povo, pacientes em geral, administração e corpo clínico, impedindo a utilização do hospital geral por portadores de hanseníase, mesmo nas intercorrências estranhas a ela. Com toda a probabilidade, a campanha exigiria pertinácia e obteria sucesso menos prolongado, consequientemente mais rápido, se objetivasse atingir os grupos apontados, na ordem inversa em que foram enumerados. Corpo clínico, administração, pacientes em geral e público apresentam-se na ordem em que podem influenciar, decrescentemente, um ao outro.

Apesar de eventuais circunstâncias poderem levar o hospital geral a participar da assistência ao paciente portador de hanseníase, pela possibilidade da caracterização de omissao de assistência, essa motivação está longe de ser o meio ideal de atıngir a colaporação desejada. Gera o subterfúgio, a reaçao. $\mathbf{E}$ um recurso herolco e, simultaneamente, paliativo, cujos resultados são antagónicos aqueles obtıaos pela educação gerada pela intormação e pela comunıcaçao.

Haveria algum processo acelerador, orientado a trazer o hospital geral ao campo da assistência do paciente portador de nanseníase! Um unico se destaca: a apiicação do enfoque sistemico à assistencia a saude; trazer o problema da hansenıase junto aos demais relacionados com a promoção da saude, englobando-os em um só contexto.

Wiener ${ }^{19,20}$, desenvolvendo a cibernética, a ciência interpretativa do controle e comunicações na maquina, homem e sociedade, lançou fundamentos essenciais para explicar o intercâmbio entre máquina, homem e sociedade e os tatores que intluenciam sua ação em direção a seus objetivos. Exemplo simples é o piloto automático das naves que, por seus mecanismos próprios, corrige a rota sempre que 
GUIMARAES, C. - Perspectivas da participação dos hospitais gerais na assistencla aos doentes de hanseniase. Rev. Saúde públ., S. Paulo, 9:401-7, 1975.

é afetada por fatos externos. Essa capacidade timoneira gerou o vocábulo cibernética, do grego "kibernetes", timoneiro. Semelhança é encontrada na capacidade dos animais superiores manterem constantes, dentro de limites determinados, as condições internas de seu organismo. Essa capacidade, denominada homeostase é um processo cibernético.

Com pouca distância cronológica de Wiener, o biólogo Bertalanffy ${ }^{4}$ concebeu a teoria dos sistemas gerais, buscando a integração em organização universal. Segundo ele, sistema é definido como "um todo complexo e organizado, uma reunião de coisas ou partes, formando um todo unitário e complexo". A alteração de qualquer das partes exige a contra-partida da adaptação das demais.

E importante distinguir os sistemas que não sofrem influência do meio que os cerca - denominados sistemas fechados daqueles que, permanentemente, estão em permuta com o ambiente onde se situam, estando continuamente influenciando e sendo influenciados.

O enfoque sistêmico disseminou-se por todas as áreas, inclusive a da saúde, tendo sido apresentado no Brasil por Chaves ${ }^{6}$.

No sistema de saúde todos os agentes e órgãos vinculados à sua assistência estão envolvidos, comprometidos e, como referido, a ação de cada um repercute nos demais e deve ser compensada.

Ao inter-relacionamento, acresce as influências externas que, pela sua ação, condicionam um reajuste permanente, constante. As influências estão em relação com o vínculo existente com os outros sistemas de que se originam pois, como a saúde, a cultura, a economia e o ambiente constituem sistemas próprios, formando todos esses sistemas correlacionados um hiper-sistema, denominado metassistema social. Um sentido mais restrito é o ecossistema, que significa o conjunto de sistemas vinculados ao sistema focalizado e que no caso da saúde incorpora, segundo Weinerman ${ }^{18}$, a educação, a as- sistência social, o controle ambiental e o emprego.

Em outro extremo situam-se os componentes do sistema: são os subsistemas. $\mathrm{Na}$ área da saúde, dependendo do ponto de fuga, são subsistemas a medicina preventiva e a curativa, a prática privada e a institucional, a saúde ambiental e os serviços pessoais de saúde; é uma miríade que se organiza caleidoscopicamente mas, no sentido objetivo, estrutura-se de acordo com a forma que convém ao sistema construído, aceito e a executar.

Essa característica de permuta de influências exige, caracteriza e é inata ao enfoque sistêmico. Daí se origina a inquietude do sistema, de sua necessidade de provocar e ser provocado, de perguntar e responder, é um permanente intercâmbio, exigindo uma metamorfose permanente. Essa labilidade só tem um compromisso: atingir o objetivo proposto. Condiciona-se no meio, a si mesmo, procurando, como Garcia, levar a mensagem adequada, medida, ajustada, eficaz e mais eficiente, buscando alta produção e, além, a produtividade.

Explanado o conceito, de forma sumária e sucinta, conclui-se, já de início, que o enfoque sistêmico exige planejamento, programação, coordenação e avaliação. Nesse contexto, abrangente e global, a assistência à hanseníase é parte de um objetivo que, quando quantificado e situado no tempo, se transforma em meta; o hospital geral passa, assim, por sua vez, a ser parte dos recursos para atingir essa meta. A avaliação dos resultados obtidos é a medida permanente para a motivação, para a manutenção ou alteração dos processos e métodos da participação de cada órgão. A avaliação constitui a vitalidade do enfoque sistêmico, pois o realimenta e revitaliza, sempre com as conseqüências de seu desempenho; é uma realimentação, "feed back", permitindo que o "kibernetes" leve, não o nau ao porto, mas o programa à sua meta.

Objetivamente, os programas, inclusive quando cuidam da assistência à hanse- 
GLIMARĀES, C. - Perspectivas da participasaão dos hospitais gerais na assistência aos doentes de hanseníase. Rer. Saúde públ., S. Paulo, 9:401-7, 1975.

níase, devem definir o papel de cada órgão e ajustá-lo quando necessário. Os vários aspectos do problema devem ser abrangidos e a responsabilidade dos órgãos, um a um, ser qualificada e quantificada.

Conseqüência lógica é a necessidade de coordenação nos diversos níveis, desde o local ao regional, estadual e mesmo nacional, se necessário, mas envolvendo órgãos preventivos e curativos, normativos e executivos visando a integração global, propósitos e ação.

0 enfoque sistêmico envolve e compromete cada órgão de "per si" e todos em geral, definindo o grau e limite de sua participação através do programa. A coordenação, qual um maestro, rege de. forma a ser uníssona a manifestação individual.

Com relação à hanseníase, assegura ele, três vantagens:

a) definição clara e precisa do grau e limite do envolvimento do hospital geral;

b) articulação do hospital geral com outros órgãos em programas que envolvem a assistência à hansenía- se. favorecendo um amplo intercâmbio de informaçōes que serão o fulcro por onde correrá a compreensão dos novos conceitos sobre a doença $e$

c) a desmarginalização da assistência à hanseníase, incorporando-a como parte integrante dos programas de saúde como uma entidade nosológica a mais e não como um corpo estranho ou diferente.

O enfoque sistêmico representa, portanto, não só a valorização da assistência ao hanseniano mas. também - e residem aí seu valor e mérito - a integração do portador de hanseníase nos programas gerais de saúde. Possibilita que ele deixe de ser o paciente para ser um paciente, mesmo parecendo, tal situação, ser um paradoxo, pois, enquanto na assistência médico-hospitalar quotidiana busca-se destacá-lo do anonimato dos assistidos, individualizando-o, busca-se aqui e neste momento de iconoclastia de velhos e falsos tabus, homogeneizar o hanseniano na multidão, para que não haja um destaque negativo, afastando-o da sociedade e dos recursos assistenciais.

RSPU-B $/ 279$

GUIMARãES, C. - [A general outlook as to the participation of general hospital's regarding attention to patients with Hansen's disease]. Rev. Saúde públ., S. Paulo, 9:401-7, 1975.

SUMMARY: A historical synthesis shous that the hospital has turned its attention towards the curative aspects of medicine. Diagnosis and therapeutic mrogress have much contributed towards this, in especial. The lazarets and their later version, the isolation hospitals, have helped in maintaining the general hospitals away, until very recently, from Hansen's disease. The medical school hospitals of São Paulo are exceptions. The importance of the education of the medical and administrative staff, the patients of the hospital, as well as the general public is stressed as being the only way of integrating assistance to the bearers of Hansen's disease in the general hospitals within a short period. A systematic approach is proposed as the solution, as Hansen's disease ought to be considered as a normal component of the general health programme. The advantages of including the patient with Hansen's disease in the general hospital's clientele in order to treat him as any other are also pointed out.

UnITERmS: Leprosy. Hospitals, general. Medical care. 
GUIMARAES, C. - Perspectivas da participaça dos hospitais gerais na assistência aos doentes de hanseníase. Rev. Saúde pübl, S. Paulo, 9:401-7, 1975.

\section{REFERENCIAS BIBLIOGRAFICAS}

1. ABEL-SMITH, B - The hospitals, 1800-1948: a study in social admmetration in England and Wales. London, Heinesmann, 1964.

2. ALTER, ' $W$ - Principes géneraux du tratament des malades a l'hopital. Nosokomeion, 4:261-2, 1933.

3. BENDER, G. A. \& THOM, R. A. - Great moments in medicne. Detroit, Mich, Farke Davis, 1961.

4. BERTALANFFY, I. von - General systems theory. New York, George Braziller, Inc., 1968.

5. CASTiglione, A. - A history of medicine. 2nd ed. New York, Alfred A. Knopf, Publ., 1947.

6. CHAVES, M. de M. - Saúde e sistemas. Rio de Janeiro, Fundasaão Getúlio vargas, 1972.

7. CONFERENCE OF THE AMERICAN HOSPITAL ASSOCIATION, 26th, Buffalo, N.Y., 1924. Transactions, Chicago, Ill., 1925. p. 414-20.

8. FRANCA, M. \& FISCHER, C. M. C. Considerações sobre a integração de um sistema coordenado de assistência médico-hospitalar. Rev. Serv. Esp. Saude pübl., Rio de Janeiro, 7: $613-9,1955$.

9. GRÃ-BRETANHA - The Labour Party. Committee on Publich Health. Memoranda, London, 1918.

10. GRA-BRETANHA - Ministry of Health. Consultative Council on Medical and Allied Services. Interin report on the future provision of medical and allied services, London, $\mathrm{H}$ is Majesty's Stationery Office, 1920.
11. GRLSCHKA, T. - The hospital as a centre of the health work in the district. Nosokomeion, 4:264-5, 1933.

12. PARISOT, J. - L'epidemiologie et la medicine preventive dans leurs rapports avec les hopitaux. Nosokomeion, 4:283-9, 1933.

13. ROSEN, G. - A history of public health. New York, M.D. Publications, 1958.

14. SARRAZ-BOURNET, M. - D'une organization hopitalière rationelle, la hierarchie dans les hopitaux. Nosokomeion, 5:13-8, 1934.

15. TERRIS, M. - Hermann Bigg's contribution to the modern concept of the health center. Bull. Hist. Med., 20: 387-412, 1946.

16. WALTER, C. W. - The aseptic treatment of wounds. New York, Macmillan Co., 1948.

17. WEBSTER's new twentieth century dictionary of English language, unbridge. 2nd ed. Cleveland, The World Publ, Co., 1969.

18. WEINERMAN, E. R. - Research on comparative health service systems. Med. Care, 9:272-90, 1971.

19. WIENER, N. - Cybernetics on control and communications in the animal and the machine. Boston, Technology Press, 1948.

20. WIENER, N. - The human use of human beings: Cybernetics and society. New York, Aron Books, 1967.

Recebido para publicaça em 10-06-75 Aprovado para publicasão em 30-06-75 